\title{
Racial Disparities in Dermatology Training: The Impact on Black Patients
}

\author{
Katherine L. Perlman, MPH; Elizabeth J. Klein, BA; Joyce H. Park, MD
}

\section{PRACTICE POINTS}

- Dermatologists should be aware of the existing health disparities in dermatology training, including lack of representation among dermatologists, treatment, patient satisfaction, and outcomes.

- Dermatologic diseases can present differently in different skin tones, and current dermatology training does not reflect these differences.

- We must continue to work toward increasing diversity of the dermatology workforce, including a diverse range of skin tones in images used in dermatology training, and teaching trainees how diseases present differently in different skin tones.
A lthough physicians commit themselves to providing equitable treatment to all patients, significant disparities remain in the dermatologic care of Black patients, who constitute $13 \%$ of the US population, which continues to grow increasingly diverse. ${ }^{1}$ Despite these changes in the population, the literature demonstrates that dermatologic training does not adequately focus on unique presentations of cutaneous pathology in the Black population., ${ }^{2,3}$ Accordingly, medical students lack proper training in how skin disorders manifest in people of color. Compounding the problem, only $3 \%$ of dermatologists are Black, creating a cultural barrier that can compromise care for Black patients. ${ }^{2,4}$ Racial disparities in dermatology training can compromise treatment, patient satisfaction, and outcomes. ${ }^{3}$

\section{Issues in Medical Education Training and Resources}

Lack of diversity in the resources used for dermatology training in medical schools affects diagnosis and treatment, as skin manifestations such as hypersensitivity reactions, rashes, and cancer can appear differently on different skin tones. ${ }^{5}$ A study of medical students' ability to diagnose common dermatologic pathologies found that when trainees were presented with photographs of dark skin, their accuracy in identifying urticaria, squamous cell carcinoma, and even atopic dermatitis was reduced, despite these diseases being more prevalent in children of African American ancestry., ${ }^{4,6}$

Dermatologic diseases also can have different distributions in different races; for example, on non-sun-exposed sites, squamous cell carcinoma in Black patients occurs at 8.5 times the frequency of White patients. ${ }^{7}$ Failure to identify diseases accurately due to insufficient training can have grave consequences for patients. Although skin cancer is less common in individuals with skin of color, it is associated with greater morbidity and mortality, in part due to delayed diagnosis. $^{7}$

Inadequate research, reporting, and instruction on dermatologic findings in patients with darker complexions further compound racial disparities in dermatology. A 2006 study of the representation of darker skin in major dermatology educational resources found that only $2 \%$ of teaching events at American Academy of Dermatology annual meetings focused on skin of color. Furthermore, the study determined that many common diseases in patients with dark skin, such as acne vulgaris and pityriasis rosea, were completely absent or limited in dermatology textbooks. ${ }^{8}$

\section{Impact on the Black Patient Experience}

Patients' therapeutic relationship with their physician also is damaged by limitations in training in diverse skin color. A study that assessed Black patients seen in a skin of color clinic (SOCC) compared to Black patients seen in a non-SOCC found that non-SOCC patients reported a lower degree of respect, dignity, understanding, and trust compared to the patients seen in a SOCC. Black patients expressed specific concerns about non-SOCC dermatologists' knowledge of abnormalities that present

Ms. Perlman and Ms. Klein are from the University of Illinois College of Medicine, Peoria, and the NYU Grossman School of Medicine, New York. Dr. Park is from the Department of Dermatology, Palo Alto Medical Foundation, Mountain View, California. 
in darker skin and Black hair. ${ }^{3}$ These findings are compounded by reports suggesting that, independent of care, structural racism contributes to dermatologic disease severity by influencing patient education level, household income, and degree of exposure to harmful environmental irritants. ${ }^{6}$

Racial disparities continue to be seen in the makeup of the universe of dermatologists and skin researchers. As of 2016, only 3\% of dermatologists were Black, making dermatology one of the least diverse medical specialties. ${ }^{2}$ Increasing the diversity of the dermatology workforce is important to improve patient satisfaction and treatment, both for minority and nonminority patients. Compared to race-discordant medical visits, race-concordant visits were shown to have a higher rate of satisfaction and better shared decision-making. ${ }^{9}$ Also, minority physicians are more likely to practice health care in areas that are traditionally underserved and to care for patients who do not have health insurance, making their participation essential in addressing some of the baseline disparities Black patients face in securing quality dermatologic care. ${ }^{1}$

\section{Structural Racism in Medicine}

Changing dermatology training to ensure improved treatment of Black patients requires not only increased attention to differences in disease presentation but also heightened awareness of underlying genetic, environmental, and structural factors that contribute to the disease course. ${ }^{6}$ For example, there is evidence suggesting that structural racism in the form of residential segregation, lower socioeconomic status, and lower educational attainment contribute to disease severity in conditions such as atopic dermatitis. There is additional evidence suggesting that White patients are more readily offered therapeutic options than Black patients. A study of racial disparities in psoriasis treatment found that Black patients with moderate to severe psoriasis were $70 \%$ less likely to receive treatment with a biologic than White patients, independent of socioeconomic factors, comorbidities, and insurance plans. ${ }^{10}$

\section{Moving Forward}

Although research continues to underscore racial disparities in dermatology, some leaders in the field are actively combating these problems. A recent study that looked at representations of dark skin images in medical educational resources found far greater representation of dark pigmented skin in web-based resources than in traditional printed texts. Specifically, the online resource VisualDx (https://www.visualdx.com/) features 28.5\% dark skin images compared to $10.3 \%$ (on average) in printed dermatology books. ${ }^{11}$ There also is increasing public awareness of these issues, with organizations such as the Skin of Color Society (http://skinofcolorsociety.org/) helping to promote interest in racial disparities in dermatology. Physicians also have created textbooks and social media accounts focused on dermatologic manifestations in skin of color. ${ }^{12}$ The Instagram account Brown Skin Matters (@brownskinmatters) has created a publicly accessible online resource where physicians and patients can see and post dermatologic diseases in skin of color. ${ }^{5}$

\section{Final Thoughts}

It is critical that physicians be trained to identify skin and hair manifestations of disease and disorders in Black patients. Training can be improved by including more images of skin manifestations in dark skin, both in medical school curricula and in new editions of dermatology textbooks. Training also must teach students about hair in Black individuals and how to properly treat it as well as related conditions of the hair and scalp. ${ }^{13}$ More research also is needed to better understand how dermatologists can improve the patient experience for Black patients. Residency programs must work to increase diversity among dermatology trainees.

Lastly, dermatology education should increasingly be supplemented with newer, web-based resources that show dermatologic manifestations across the spectrum of skin tones. Dermatology training must be adapted to better account for diverse patient populations and increase its focus on the systems that produce baseline disparities in disease morbidity and mortality.

\section{REFERENCES}

1. Pandya AG, Alexis AF, Berger TG, et al. Increasing racial and ethnic diversity in dermatology: a call to action. J Am Acad Dermatol. 2016;74:584-587.

2. Gallegos A. Dermatology lacks diversity. Dermatology News. June 1, 2016. Accessed November 18, 2020. https://www.mdedge.com/dermatology /article/108920/practice-management/dermatology-lacks-diversity.

3. Gorbatenko-Roth K, Prose N, Kundu RV, et al. Assessment of black patients' perception of their dermatology care. JAMA Dermatol. 2019;155:1129-1134.

4. Fenton A, Elliott E, Shahbandi A, et al. Medical students' ability to diagnose common dermatologic conditions in skin of color. J Am Acad Dermatol. 2020;83:957-958.

5. Prichep D. Diagnostic gaps: skin comes in many shades and so do rashes. NPR website. November 14, 2019. Accessed November 19, 2020. https://www.npr.org/sections/health-shots/2019/11/04/774910915 /diagnostic-gaps-skin-comes-in-many-shades-and-so-do-rashes.

6. Tackett KJ, Jenkins F, Morrell DS, et al. Structural racism and its influence on the severity of atopic dermatitis in African American children. Pediatr Dermatol. 2020;37:142-146.

7. Gloster HM, Neal K. Skin cancer in skin of color. J Am Acad Dermatol. 2006;55:741-760

8. Ebede T, Papier A. Disparities in dermatology educational resources. J Am Acad Dermatol. 2006;55:687-690.

9. Cooper LA, Roter DL, Johnson RL, et al. Patient-centered communication, ratings of care, and concordance of patient and physician race. Ann Intern Med. 2003;139:907-915.

10. Takeshita J, Eriksen WT, Raziano VT, et al. Racial differences in perceptions of psoriasis therapies: implications for racial disparities in psoriasis treatment. J Invest Dermatol. 2019;139:1672-1679.e1.

11. Alvarado SM, Feng H. Representation of dark skin images of common dermatologic conditions in educational resources: a cross-sectional analysis [published online June 18, 2020]. J Am Acad Dermatol. doi:10.1016/j. jaad.2020.06.041.

12. Rabin RC. Dermatology has a problem with skin color. The New York Times. August 30, 2020. http://www.nytimes.com/2020/08/30/health /skin-diseases-black-hispanic.html. Accessed November 19, 2020.

13. Bosley RE, Daveluy S. A primer to natural hair care practices in black patients. Cutis. 2015;95:78-80. 